\title{
Spatial process and data models: Toward integration of agent-based models and GIS
}

\author{
Daniel G. Brown ${ }^{1,3}$, Rick Riolo ${ }^{3}$, Derek T. Robinson ${ }^{1,3}$, \\ Michael North ${ }^{2}$, and William Rand ${ }^{3}$ \\ ${ }^{1}$ School of Natural Resources and Environment, University of Michigan, 430 E. University, \\ Ann Arbor, MI 48109-1115,USA (e-mail: danbrown@umich.edu) \\ ${ }^{2}$ Argonne National Laboratory \\ ${ }^{3}$ Center for the Study of Complex Systems, University of Michigan
}

Received: 13 November 2003 / Accepted: 25 October 2004

\begin{abstract}
The use of object-orientation for both spatial data and spatial process models facilitates their integration, which can allow exploration and explanation of spatial-temporal phenomena. In order to better understand how tight coupling might proceed and to evaluate the possible functional and efficiency gains from such a tight coupling, we identify four key relationships affecting how geographic data (fields and objects) and agent-based process models can interact: identity, causal, temporal and topological. We discuss approaches to implementing tight integration, focusing on a middleware approach that links existing GIS and ABM development platforms, and illustrate the need and approaches with example agent-based models.
\end{abstract}

Key words: Object-orientation, Agent-based models, Spatial-temporal modeling, Topology

\section{Introduction}

This paper addresses the representation of both form and process, as spatial data models and spatial process models, respectively, and how relationships between these representations might be structured to better facilitate scientific inquiry and application. Raper and Livingstone (1995)

The authors acknowledge financial support from the U.S. National Science Foundation under the Biocomplexity in the Environment Program, Coupled Human-Natural Systems, grant \# BCS-0119804. Pam Sydelko of Argonne National Laboratory contributed greatly to the description of the Red-Cockaded Woodpecker Model. The Center for the Study of Complex Systems at the Univesity of Michigan provided computer resources and other support. Daniel Miller, also of Argonne National Laboratory, contributed to the description of the Infrastructure SymSuite. Argonne National Laboratory, a U.S. Department of Energy Office of Science laboratory, is operated by The University of Chicago under contract W-31-109-Eng-38. We thank Kevin Johnston at ESRI for his support and encouragement. 
addressed this same issue in the context of geomorphological processes and phenomena. Their definitions of process and data models suffice for our purposes. "Process models generally express theories predicting the nature of the exchange of energy and mass within systems, over time." (Raper and Livingstone 1995; P. 361). In a broader context, this definition could be expanded to include exchange of ideas, culture, viruses, etc. "By contrast, data models express theories predicting the structure of the realworld domains in terms of entities and their attributes organized in interrelated sets. One of the basic problems encountered when 'coupling' environmental models with GIS is that the former are specified as process models while the latter are specified as data models" (Raper and Livingstone 1995; P. 361). In addition, they argue that progress in object-oriented data modeling presents a tremendous opportunity for coupling with object-oriented process models. This is an argument we pursue here.

Geographic information systems (GIS) use multiple spatial data models for representing and storing information about phenomena with spatial location and/or extent (Lo and Yeung 2002). The focus of data models in GIS has been on spatial, at the expense of temporal, dimensions (Peuquet 2002). Space and time can be defined in absolute or relative terms (Blaut 1961; Couclelis 1997; Wachowicz 1999; Goodchild 2002). Whereas absolute space and time offer a rigid geometric structure within which phenomena are referenced, the relative view references location largely in terms of relationships, topological and temporal, between features (Peuquet 2002). Object-oriented data models, which have grown increasingly sophisticated in GIS (Leung et al. 1999), assign location as an attribute of features rather than of the space itself, and allow representation of relative space. In contrast to GIS, process models make use of sophisticated representations of time and behavior, often at the expense of sophisticated representations of space and spatial relationships. By assigning temporal behavior as an attribute of objects rather than of the environment, object-oriented process models (e.g., individual-based and agent-based models) allow for a relative view of time, in which objects are updated asynchronously (Westervelt and Hopkins 1999), as opposed to updating all at once (i.e., synchronously).

In this paper we address the coupling of GIS-based data models with agent-based process models. After reviewing fundamental types of data and process models and implications for their coupling, we review the successes achieved in coupling synchronous models of change with raster-based GIS. Next, we identify the largely unrealized opportunities for coupling objectbased spatial process models with multiple GIS data models. We describe four key relationships that affect the interactions between geographic data (fields and objects) and agent-based (i.e., object-oriented) process models. We describe some alternative approaches to implementing GIS-ABM integration, focusing on an approach that involves development of middleware to manage connections between agents and spatial features, and some implementation issues. Then, the relationships and some of the implementation approaches are illustrated by describing four models that make use of them in common combinations. 


\section{Spatial data models}

Spatial data models describe two fundamentally different conceptions of space. The field view represents space as a continuously varying distribution of geographic variables, and the raster data model is often used to approximate this view by discretizing an absolute space and subdividing it in regular intervals. In contrast, the object view focuses on discrete entities, which have location, some level of spatial extension and attributes, and are usually represented as spatial features (i.e., points, lines or polygons) using a vector data model. Though many phenomena can be reasonably represented as either fields or objects, some geographic phenomenon have both field and object characteristics, and might require a combination of these views. One example is an aggregation of many discrete objects, created as outputs from an optimization algorithm, that represents a solution space as a field of objects (Cova and Goodchild 2002). Alternatively, an object, like a storm cell, can exhibit continuous spatial variation (e.g., wind speed) within its spatial extent (Yuan 2001).

Object-oriented data models, which make use of hierarchically nested entity definitions that encapsulate behaviors (e.g., about allowable entries in the database) and inherit data characteristics from higher levels, have become mainstream within commercial GIS, for example in the form of the geodatabase structure within ArcGIS (ESRI 2004). In addition to facilitating a closer conceptual relationship between features in the database and discernible entities in the real-world, object-orientation also facilitates the implementation of relative space. One way to represent relative location using object-oriented GIS is through topological rules that can proscribe certain kinds of spatial relationships and require others. For example, a topological rule can specify that a spatial feature in one layer (e.g., a house) may not overlap a spatial feature in another layer (e.g., a lake). These topological rules describe certain logical characteristics of the physical world that can prevent erroneous placement of features.

Several researchers have proposed extensions to existing spatial data models to provide richer representations of time. These extensions have used (a) spatio-temporal objects to represent evolution of the geometry, properties, and location of entities through time (Worboys 1994), (b) event sequences on a grid to keep track of how a process evolves (Peuquet and Duan 1995), (c) events and processes to describe spatio-temporal phenomena that possess both field and object characteristics (Yuan 2001) and (d) versioning, through the use of "amendment vectors," to represent boundary changes (Langran 1992). By explicitly including time, these extended spatialtemporal data models facilitate visualization and analysis of dynamic attributes and features defined with spatial and temporal extension. However, even with inclusion of temporal referencing or time-based attributes, these data models have not (with few exceptions; Raper and Livingstone 1995) included explicit representations of the processes (i.e., sequences of operations) by which spatial features change and move, focusing instead on representing the changing structure of the phenomena. Representations of process are important, however, in explaining why the structures change as they do and are also required if analytical operations are to be implemented across space and time, for example if we desire to 
interpolate features and/or attributes across time. Such representations of process, then, interact with and extend the capabilities of spatio-temporal data models.

\section{Spatial process models}

A variety of approaches exist to represent dynamics in process-based models. We borrow, in a general sense, two conceptual views of spatial processes from the Eulerian and Lagrangian views of fluid dynamics. The Eulerian view describes the processes that influence properties (e.g., temperature) at fixed locations, and thus is a description of change. The Lagrangian perspective, on the other hand, tracks the changing location of particles through space and, therefore, is a description of movement. Many processes in the real world could be reasonably described as either Eulerian or Lagrangian. For example, as a cold air mass moves (described as Lagrangian motion) over a location it causes the temperature at that location to drop, affecting an Eulerian change. The duality between models of change versus movement can be extended to describe nearly any geographic process, and the choice of which approach to take for representing any given process is made on the basis of both conceptual and practical considerations. Here, we describe how change and movement models interact differently with spatial data models.

\subsection{GIS-based change models}

Perhaps the simplest models of change are based on the Markov process, in which the state of a random variable at a location in the future is only dependent on its current state (Sheynin 1988). For example, many early computer simulations of land-use change were based on Markov formulations (Burnham 1973, Bell 1974). A variety of cellular models have enhanced these Markov formulations by incorporating spatial interactions into the calculation of probabilities of change at locations (e.g. Verburg et al. 1999; Brown et al. 2000). For example, to investigate diffusion of innovation and its effects on regional-level behavior and spatial patterns, Hägerstrand (1967) developed cellular models using local interaction rules. The probabilities of future states were based on the surrounding states within some window. Tobler's $(1970 ; 1975 ; 1979)$ "cellular geography" built on Hägerstrand's approach, and perhaps on the work of Burks (1970) who was a contemporary of Tobler's at the University of Michigan, to model change in a variety of systems, including urban development in Detroit (Batty 1997). The work of Burks (1970), his mentor von Neumann (1966), and others (Ulam 1962) resulted in the development of cellular automata (CA), which use simple rules to represent complex dynamics resulting from social, physical, biological and computational processes (Toffoli and Margolus 1987). In their purest form, CA represent change on a lattice deterministically, using rules that relate the current state at a location and at immediately surrounding locations with the next state at that location.

The logic of integrating field-based spatial data models with change-based spatial process models is strong (Kemp 1997) and, for this reason, has been exploited in a variety of GIS. Eastman (2001) introduced into a recent 
version of Idrisi, a raster GIS system, a spatial modeling component that combines a Markov process representation of state changes with a cellular model to introduce spatial interactions. In addition, Idrisi now includes a graphical modeling environment, in which the "map algebra" functions common in raster GIS (Tomlin 1990) can be applied iteratively to maps in discrete time steps to implement change processes. It is relatively simple, for example, to employ spatial filtering and map reclass operations iteratively to implement simple cellular automata within this environment. Burrough and colleagues at Utrecht University (Van Duersen 1995; Wesseling et al. 1996) developed a system, called PCRaster, that extends the map algebra to include representations of time and dynamics. The system maintains a timer to track the progression of time in discrete steps, and includes explicit representation of change and transport processes.

Discrete-state spatial models of change processes operate on a discrete representation of continuous space and employ discrete time steps and synchronous updating to represent time. Models of flow and transport processes based on fluid mechanics, such as those used to represent hydrological flow and atmospheric processes, are based on continuous notions of space and time (e.g., through the use of differential equations). However, their implementation with GIS usually involves a discretization of space and time similar to the discrete state models above (Maidment 1996). The processes in this discretized environment are commonly represented as finite difference or finite element models, which essentially represent the future state at a location, in terms of the quantity or flux of the fluid or materials, as a function of its current state and flux and its surrounding states and fluxes. These common discrete numerical solutions create fluid models that interact with spatial data models in very similar ways to the discretestate models.

\subsection{GIS-based movement models}

The most common GIS-based movement models involve models of flow and transport, e.g., hydrological flow and transport of pollutants. However, while flow and transport models represent the dynamics of movement, the underlying implementation is usually expressed in terms of updating (or changing) states, which can include fluxes, at fixed locations and over discrete time steps. Similarly, though CA and other cellular models focus on modeling changes in state, emergent features can appear to "move" in space. Conway, in developing his "Game of Life" illustrated the emergence of moving features (i.e. "gliders") on the basis of simple local change rules (Gardner 1971). Fire is another emergent feature that seems to spread across a landscape as a result of the interactions encoded in the individual state changes (Clarke et al. 1994). So, by explicitly representing how states change at a location, some types of movement can be captured implicitly. An important limitation of this approach, however, is that geographic features (e.g., in an object-based spatial data model) are not represented explicitly in the model. Moreover, the movement is implicit in the change rules, and cannot be queried directly. As a result, the relationship between the process as observed in the real-world (i.e., movement) and its representation in the model is not always clear. 
GIS, in general, do not include operators that explicitly describe movement of features (De Vasconcelos et al. 2002; Westervelt and Hopkins 1999). For this reason, it is relatively more difficult to implement object-oriented movement models than change models in close coupling with spatial data. However, such coupling is a logical next step in merging spatial data with spatial process representations.

\subsection{Agent-based models and object-based movement}

Object-oriented process models, which include individual-based models (IBM) commonly used in ecology (DeAngelis and Gross 1992) and agent-based models (ABM) common in the social sciences (Epstein and Axtell 1996), allow for modeling movement and have developed independently of GIS. The object-oriented framework of ABM involves identification of agents and of a temporal framework within which those agents perform actions. While many different types of agents can exist, the following general definition is common: an agent is a self-directed object, i.e., it has the ability to satisfy internal goals or objectives through actions and decisions based on a set of internal rules or strategies (Iglesias et al. 1999). These agents may be dynamic in either state (i.e., change) or space (i.e., movement) and may, through their actions, change the state or location of other objects, processes, or environments around them. Agent dynamics are most naturally implemented in an ABM by a set of behaviors ("methods") that can include conditional decision making and other (nonlinear) rules that distinguish them from mathematically continuous models (Parunak et al., 1998). The ability for Lagrangian motion (i.e., agent movement) distinguishes ABM and other object-oriented modeling frameworks from the change-based spatial models described above. It also creates additional challenges for integrating these models with GIS, as described in more detail below.

ABM dynamics are defined at the level of (a) agent behaviors that result in change and movement, and (b) the independent dynamics, if any, of nonagent objects. Thus to represent dynamics, ABMs are implemented as discrete event simulations (Zeigler et al. 2000), in which some kind of "scheduling" mechanism handles the sequencing of agent behaviors and events. An ABM may implement scheduled events in three ways:

- Events may be sequenced in a synchronous step-wise fashion. For example, each agent, set of agents or non-agent object is signaled to perform its tasks once at each time step or once every $n$ time steps.

- An event may be scheduled to occur only once at some time step $n$. Any number of different events may be scheduled to occur in this fashion providing a predetermined history of events to take place.

- The model may encapsulate 'event-driven' processes whereby model agents may trigger events to occur or may add events to the schedule or queue of events to take place (Ropella et al. 2002).

The choice of both an event-scheduling approach and a temporal resolution (i.e., frequency of time steps) can have serious consequences for the behavior of the model (Liu and Andersson 2004). Though more research 
is surely needed on the consequences of these choices, they make it clear that ABMs have significant flexibility with respect to their handling of time.

On the other hand, ABMs often use relatively limited representations of space. For example, ABMs frequently use hypothetical spaces based on square or hexagonal tessellations, and only recently have ABMs begun to use real-world spatial data. To avoid edge effects on the performance of some models, researchers commonly use a toroidal representation of space, which wraps around from top-bottom, left-right, and vice versa. The rich temporal representations (agents and processes) of agent-based models, therefore complement the spatial data representations (fields, objects and functions) of GIS. The object-oriented nature of both presents tremendous opportunities for their integration.

\section{Relationships affecting process-data coupling}

Given the complementarities of spatial data models (fields and objects) and agent-based (i.e., object-oriented) process models, and their combined potential to improve on integrated representations of spatial patterns and temporal processes, we argue that tight coupling of models and data within $\mathrm{ABM}$ and GIS, respectively, can reap benefits in terms of both efficiency, through reduced computing times, and capability, through new functionality. Attempts to integrate ABM and GIS techniques have raised several conceptual and technical questions (Gimblett 2002). These issues broadly fall into questions of ontology and process, i.e., how are entities and processes represented, and how do those representations interact, respectively. As an instance of the former, Bian (2003) concluded that the environment within an individual-based model can be represented as either patch-based (i.e., objectbased), maintaining object-orientation in both the model and data, or fieldbased, such that object-oriented individuals interact with a discretized environment of attributes. She discounts the value of treating all cells in a grid-based environment as objects on both technical (i.e., due to inefficiencies) and ontological (i.e., poor match to conceptual view of fields) grounds.

More generally, developing models that make use of both GIS and ABM techniques requires the specification and implementation of relationships between agent-level processes and spatial data. In order to facilitate better understanding of the technical and conceptual issues that can arise when creating models that tightly couple GIS and ABM techniques, we present below a description of four key relationships that affect the integration of a dynamic GIS data base and an agent-based process model (Fig. 1). We describe how the relationships affect representations of dynamic geographical systems with the goal of better understanding how ABM and GIS techniques can be coupled to treat spatial and temporal dynamics equally well. Approaches to implementing these relationships are taken up in a later section.

\subsection{Identity Relationships (Fig. 1a)}

By defining an identity relationship between an agent and a spatial feature or features, GIS techniques can be used to store the geographic extent and 


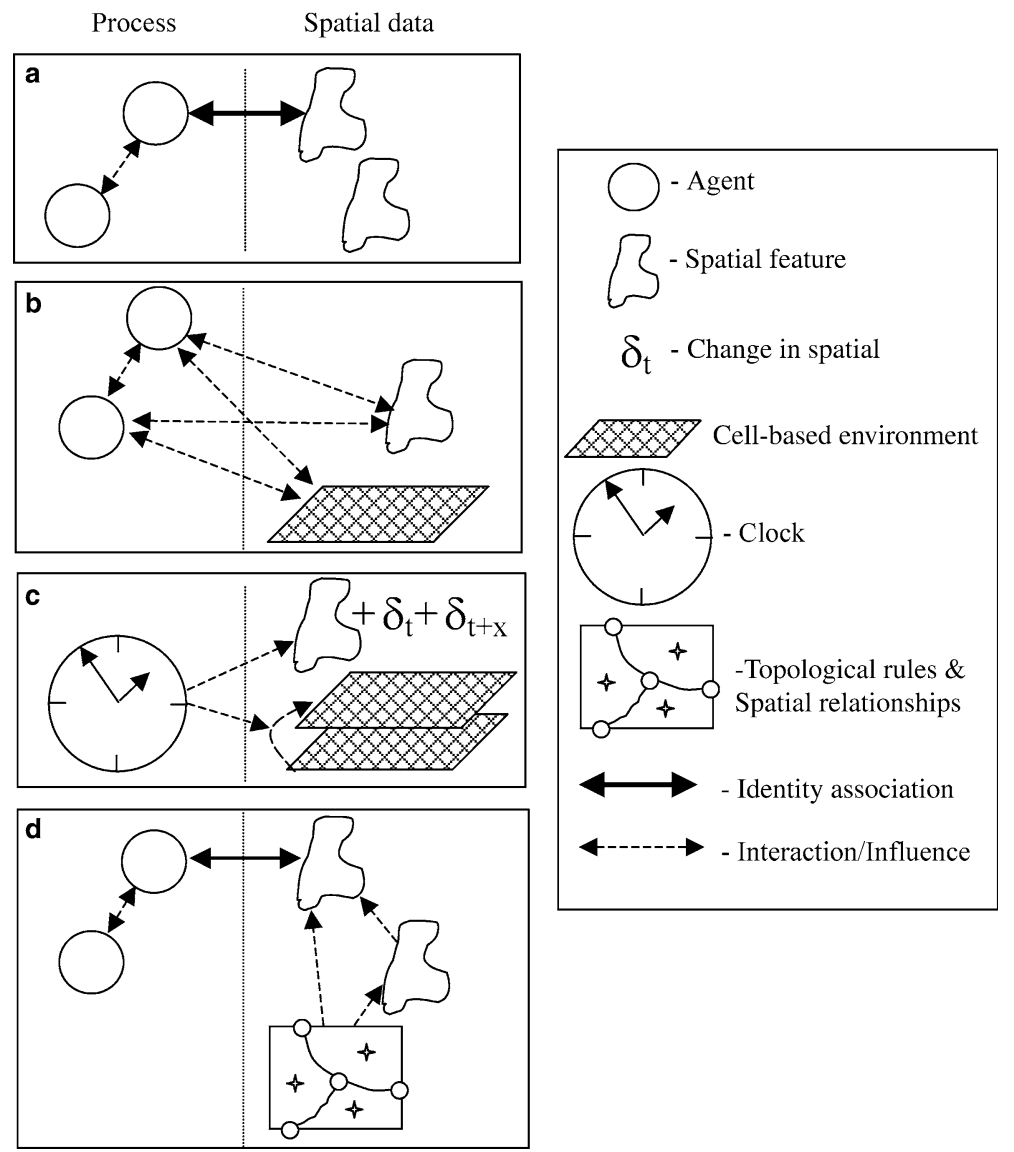

Fig. 1. Conceptual illustration of four key relationships that affect the interaction of spatial data models and their associated techniques and agent-based process models and their associated techniques. The four relationships are (a) Identity Relationships, (b) Causal Relationships, (c) Temporal Relationships, and (d) Topological Relationships. The graphic portrays the relationships conceptually and is not meant to describe any particular implementation

attributes of the feature, while $\mathrm{ABM}$ techniques represent the behavior of the agent and the change in associated feature(s). Thus (a) spatial features associated with agents can move or change, and (b) attributes of features associated with agents can change. The spatial features that are associated with agents can be stored in the GIS as polygons, lines, points, or cells in a raster. It is possible for an agent to be associated with only one spatial feature (i.e., a one-to-one relationship) or more than one spatial feature (i.e., a one-to-many relationship). In any given model, there also can be some spatial features that are not related to agents and some agents that are not related to spatial features. One example of an identity association is vehicles represented as agents, with movement rules, that are associated with points and attributes in a spatial database (Deadman and Gimblett 1994). The points move when the vehicles move and the attributes of the points change as the vehicles change (e.g., their fuel levels change). As a given model 
progresses, the agents might update their own locations, shapes, and/or attributes, affecting changes in a spatial database and/or graphic display. The behavior of an agent can interact with (i.e., affect or be affected by) other agents, whether or not they have associated spatial features. For some types of movement or spatial interactions, however, it might be necessary to compute the spatial relationships among features (see item 4 below).

\subsection{Causal relationships (Fig. $1 b$ )}

In many models, agents have the ability to take actions that affect spatial features and/or their attributes, even if there is no identity association between the agent and the spatial feature(s) it is acting on (i.e., non-agent features). Agents can take actions that result in changed locations or attributes of features, or they can take actions that change the values of an attribute on a field (e.g., a raster). For example, a public health agency (i.e., agent) could implement a disease control strategy that involves treating wetlands (i.e., polygon feature) with mosquitocides or draining them to reduce the prevalence of mosquito-borne diseases. The actions of the agency affect the attributes (i.e., mosquito abundance) and/or spatial expression (i.e., through draining) of the polygon features representing the wetlands. Note that the behavior of other agents, e.g., nearby households, might be subsequently affected by the changes in the spatial features, making appropriate handling of temporal dynamics important.

\subsection{Temporal relationships (Fig. 1c)}

Two types of actions in a coupled process-data model are fundamentally time-sensitive: (a) the actions of the agents and (b) the updating of attributes or locations of features in a database or display. Either can be handled using synchronous or asynchronous approaches. When actions are handled synchronously, the coordination of these dynamics is relatively straightforward. All agent actions can be carried out at once, and changes to features and attributes are written to the database (and to the display) at the same instance. (Note: In non-parallel computer implementations, such models carry out the changes in series, but they represent processes that are instantaneous.) To record the history of the process, the model might create a new attribute, set of spatial features, or raster layer, which is saved to a database along with some indicator of the time. There is little value in using asynchronous updates to a database, when the model runs synchronously, because the model does not change the features or attributes between discrete time steps. However, synchronous database updates can be made less frequently than synchronous model time steps.

When agent actions are asynchronous, database updates can be made either synchronously or asynchronously. When handled synchronously, database updates reflect snapshots of a model run taken at regular time intervals and are made in a similar manner as a synchronous model, described above. However, asynchronous model actions used together with synchronous database updates can complicate issues associated with the "speed of light" problem. The problem is, if an agent requests a change in 
one part of the world, how soon are other agents (or non-agent objects) located in other parts of the world affected by that change? Exactly how to handle this issue depends on the specifics of what is being modeled, the time scales required, and the modeling goals. This problem is especially complicated when agent actions are dependent on the database to provide information about the state of the world at a given time, because of the delay in updating a given part of the database associated with the specified synchronous-updating interval. An alternative approach is to use asynchronous database updates to mirror the asynchronous agent actions. This implies an alternative database structure, requiring that all changes to features or attributes are stored explicitly (e.g., to create different versions of features) along with time stamps to indicate when these changes occurred.

Further complicating the issue of temporal relationships are models in which there are dynamic processes that are, more or less, independent of the dynamic processes represented by agents (e.g. Westervelt and Hopkins 1999). For example, if a process of natural plant succession using a Markov process is updating a landscape while agents move across it, then some coordination of the timing of updates to the Markov process and the movement of agents is needed. To coordinate these processes, the clock that governs agent behavior must be coordinated with the clock that governs the updates of the Markov model.

\subsection{Topological relationships (Fig. 1d)}

Movement of spatial features, either by processes internal to their associated agents or by those of other agents (only the former are illustrated in Fig. 1d for simplicity), can require basic information about the physical world or spatial relationships between features. Whether a particular move is desirable or physically possible can require information about (a) topological rules specified for a set of spatial features in relation either to each other or to another set of spatial features, or (b) the spatial associations between features as determined by calculations of distance, cost of interaction, or visibility. For example, movement of a vehicle to a particular location may not be possible because a building or another vehicle already occupies that location. Alternatively, a person (i.e., agent) may be programmed to move to a nearby location on the landscape that maximizes some spatially defined objective, like visibility.

Clearly, models make use of the relationships described above to varying degrees. However, identifying the general types of relationships that are possible can move us toward general concepts and tools for linking process and data models. Next, we discuss the implementation of these linkages.

\section{Software implementation}

Where real-world spatial data are to be used by an ABM, some form of coupling with GIS data and functionality is necessary in order to create models that effectively represent both complex spatial structures and rich dynamical processes. The use of loose coupling, which involves passing interchange files between the model and the database, limits models in a 
variety of ways. First, computational efficiency is low because of the costs of input and output. Because many models are very computationally demanding, this is an important issue. Secondly, loose coupling precludes direct use of existing database query and analysis functions within the models (Gimblett 2002) and direct use of modeling functions with the database. For these reasons, more tightly coupled models are desirable. While tighter coupling provides many advantages, such coupling also raises a number of implementation issues, which we discuss next.

Implementing tightly coupled process-data models requires choosing an appropriate architectural platform. Initial attempts to tightly couple ABM and GIS functionality (several of which are described in the Examples section) have taken an ABM-centric approach. One of these approaches has involved the use of software libraries of GIS functions within ABMs and the encapsulation of features and attributes with the agents, where there are identity relationships. For example, the GeoTools Java library (http:// www.geotools.org), which includes GIS data management and visualization functionality, is available for use by models developed with the RePast ABM development platform (University of Chicago 2003). It is also possible to implement (i.e., write code for) spatial data management and analysis functions within a model. However, the costs of this strategy increase dramatically as a model's use of frequent updates to spatial data and complicated spatial analysis functions grow. Functions that are readily available within the GIS framework need to be written, debugged, tested, and documented within the ABM framework; thus the development time increases. Also, code developed in the ABM to perform GIS-like tasks may not be as efficient as that within a GIS. This is partly because ABM platforms are not built to handle primarily spatial interactions, but also because GIS systems have developed over many years with attention to increased efficiency. Additionally, use of standard GIS tools for spatial analysis improves functional transparency of a model, as the model makes use or well-known and understood algorithms.

A GIS-centric approach to coupling, i.e., implementing ABM functions and models within a GIS system and user interface, is an attractive alternative. A GIS-centric coupling would allow such models to run interactively within the graphical user interface of a GIS package. Given the large GIS user base, such accessibility could expand the potential user base for any given model. A complement of the ABM-centric approach, GIScentric coupling can be carried out using software libraries of ABM functions accessed through the GIS interface. To fully embed ABMs within a GIS would require the ability to encapsulate a wide range of agent behaviors with spatial features and a means of keeping and coordinating time. The obvious difficulties of this approach arise because GIS do not usually provide these capabilities. They therefore lack the most basic tools required for agentbased model creation and use. We know of no existing implementation of an ABM embedded completely within a GIS environment.

\subsection{Integrated systems}

A third broad approach to integration is centered on neither ABM nor GIS, but makes use of the functionality available in both environments. One 


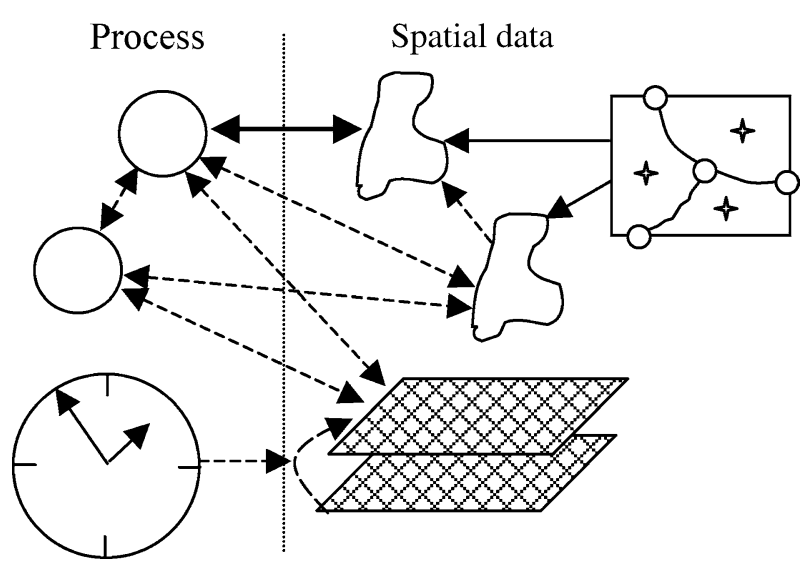

Fig. 2. A composite diagram showing all relationships between process models and spatial data models as they are displayed separately in Fig 1 (legend is the same)

alternative is to build such an integrated system from the ground up. For example, Torrens and Benenson (In Press) argue for the implementation of geographic automata systems (GAS), which tightly couple spatial data and process models within a single, integrated system. They demonstrate the Object Based Environment for Urban Systems (OBEUS), which is a model development environment, like RePast (Collier 2000) and Swarm (Minar et al. 1996), but with particular emphasis on spatial interactions.

In contrast to building a completely new system from the ground up, an intermediate "middleware" alternative builds on existing platforms and involves the development of software to handle the identity and causal relationships between the agents within an $\mathrm{ABM}$ environment and spatial features within a GIS environment, as well as the temporal and topological relationship issues that arise in the model. Such middleware could allow a model to make use of the tools that are most appropriate for a given task. Rather than providing all functionality within one system, the middleware manages connections between systems, so that the relationships described above can be implemented. An important advantage of this approach is that models can both be developed using an ABM platform and run and visualized within a GIS platform. It also takes advantage of the substantial investments in developing existing GIS and ABM environments. Although this approach does not take full advantage of the ontological and practical benefits of encapsulation within an object-oriented framework, the fact that both ABM and GIS are object-oriented makes the coupling possible. Further, we see this as a practical direction for short- to medium-term development, because the approach requires only linking existing systems, rather than building new ones.

A potential disadvantage of creating a model that relies on both GIS and ABM toolsets is increased start-up costs, because of the added complexity required to handle both software environments and their integration. It is important to avoid the temptation to use tools that are more sophisticated than necessary for a given model. For example, if a model requires only minimal spatial analysis and interaction with real spatial data, tight coupling may be unnecessary. Another disadvantage can be the dependence of a 
model on two toolsets instead of one. A model is more likely to suffer obsolescence because of changes in either the GIS or ABM platforms. Platform dependence might also turn off potential users who do not have or like one or both of the choices of platforms.

An example of this approach, implemented to link RePast and ArcGIS, is illustrated in the Infrastructure SymSuite example described below. The model developer simply names a class of agents to match the name of a feature layer within ArcGIS, and names agent properties to match the names of attributes in the layer. When the model is run, the middleware creates an agent for each feature and sets the agent properties according to the feature attributes. Changes in agent properties also cause updates to the attributes of GIS features.

\subsection{Handling the four relationships through middleware}

Implementing a middleware approach between ABM and GIS first requires establishment of identity relationships between agents and features. To represent identity relationships, a software mapping is required to identify object ID(s) in the GIS database associated with each agent, and vice versa. As agents perform actions, they implement a change, or send messages to the GIS software that requests a change, in the location and/or attributes of the spatial features that are written to a graphical output and/or to the database.

To implement causal relationships, in which the state of the GIS affects the behavior of agents and the behavior of the agents may affect the state of the GIS, agents in the ABM query the GIS for objects (some of which might be associated with other agents) with particular locational or attribute characteristics and may affect changes to their location or attributes.

Because ABM development platforms (like Swarm and RePast) have the potential for richer representations of time, and have better mechanisms for implementing dynamical processes and relationships at various time-scales, coordination of temporal relationships should be managed by the common temporal reference maintained by the ABM. This can be implemented by either directly requesting the GIS to carry out updates at particular (ABM) time steps, or by communicating time-step references to the GIS as needed. The simplest cases are those in which all dynamics are represented in the $\mathrm{ABM}$, and the GIS is primarily used as a database and graphic display tool. One way to implement coordination of time, when the GIS platform includes an autonomous process model (like the Markov model described in the discussion of temporal relationships) is to have the ABM send a message to the GIS indicating that so many ticks on the ABM clock have elapsed and that it is time for the GIS model to advance and update the landscape maps. This, of course, requires careful coordination of the meaning of time steps.

Because GIS have more sophisticated representations of space, topology, and spatial relationships, the topological relationships should be handled within the GIS. Communicating topological and spatial relationships such that they provide constraints on agent behavior could be implemented by (a) having agents query the GIS each time a move is attempted, or (b) having agents that want to move act by sending a "try to move" message to the GIS, for example using feature editing functions. In the latter case, the GIS could 
respond in turn by either moving the agent as requested (and thus updating the GIS database and associated graphical displays), or by returning a message to the agent about why the move could not be executed (e.g., the location was already occupied).

\section{Examples}

Here we describe several models to illustrate interactions between spatial data and spatial processes and, therefore, the different requirements for integrating ABM and GIS functionality. The specific cases were selected to reflect commonly encountered types of models. These examples demonstrate multiple types of models, in terms of the four relationships listed above, and implementation strategies. Focusing on the middleware development approach used in the last example, we address future developments needed to improve on the existing implementation.

\subsection{Urban land-use change}

In a project on land-use change at the urban-rural fringe, called SLUCE (http://www.cscs.umich.edu/sluce), we are studying how individual decisionmaking drives land-use decisions that affect and are affected by environmental systems. The investigation utilizes an ABM called SOME (SLUCE's Original Model for Exploration) to model the residential location decision process (Rand et al. 2003; Brown et al. 2005). This model incorporates two kinds of agents: homebuyers and service centers. It also incorporates several spatial data layers that characterize aesthetic quality, roads, and locations of current development.

Agents have no identity relationships with spatial features in this model, though the service centers and homebuyers do participate in the land-change processes. The primary relationships that exist in this model are causal. The agents affect change in attributes on a grid-based landscape and their decisions are affected by the attribute values on that landscape. The residents make decisions about where to locate based on features such as aesthetic quality, distance to service centers, and nearby density. Service centers are programmed to enter at specified intervals and to locate near recent residential development. Thus, homebuyers are affected by environmental qualities and then influence the behavior of the service centers, which in turn influences the behavior of future homebuyers by changing the distances to service centers. This model uses the simplest class of temporal relationships, i.e., all agents entering the system each time step perform their actions and the $\mathrm{ABM}$ updates the raster database before the next time step begins. Topological relationships include checking if development is prohibited at a location because other agents have already developed it or because development is not possible, e.g. lakes or greenbelts. Also, nearby density of a given cell is calculated using a kernel calculation of the number of developments around each cell, and distance to service centers is calculated for each cell.

The existing implementation of this model uses elements of both loose and ABM-centric coupling. The grids of initial conditions are read from interchanges file that are produced by the GIS. Results from the ABM, 

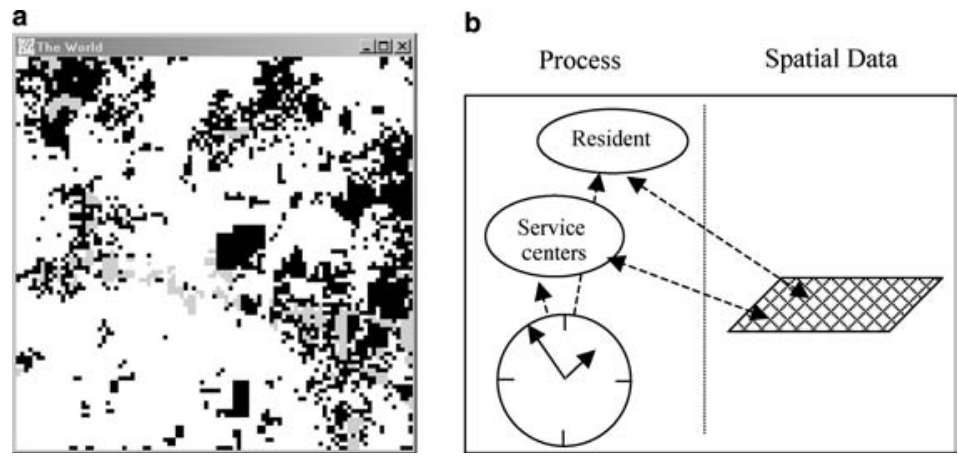

Fig. 3. The SOME model. a Map showing cells that are occupied by residents (black) and service centers (gray) at a particular time during the run of the SOME model. b Conceptual relationships between process and spatial data models
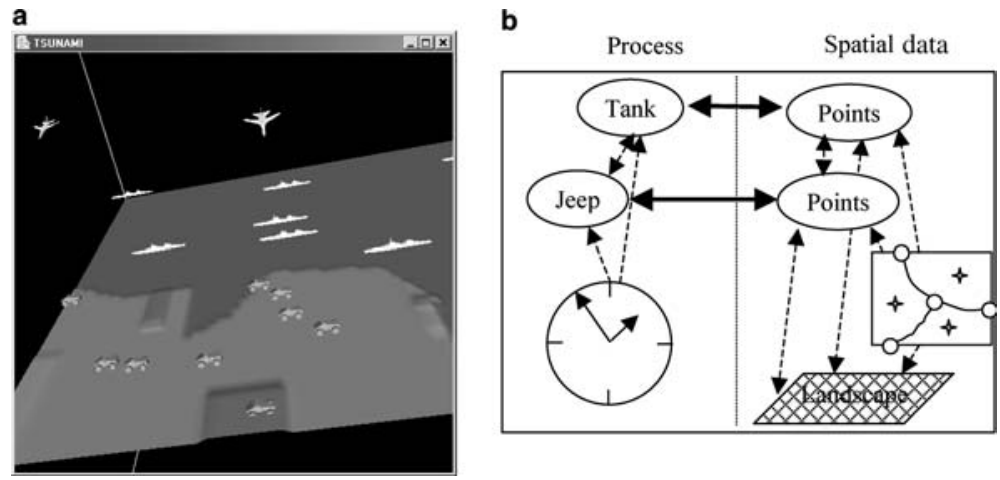

Fig. 4. The TSUNAMI Model. a Local battle space visualization with geography and agent locations. The agent locations and geography are for illustrative purposes only. b Conceptual relationships between process and spatial data models. Only jeeps and tanks are shown for illustration, though other agents are also present

i.e., maps showing cells occupied by residents and service centers (Fig. 3a), are then written to the same interchange format that can be read into the GIS for viewing and analysis. Functions written within the model are used to handle the spatial data, including special functions written to carry out spatial analyses (e.g., window operations and checking for occupied locations, and distance calculations). Because of the relatively simple types of interaction between the model and the database (Fig. 3b), the biggest benefits of tighter integration would come from enhanced availability of spatial analysis functionality (from the GIS) within the model.

\subsection{Military mobile communications}

The goal of the Tactical Sensor and Ubiquitous Network Agent-Modeling Initiative (TSUNAMI) is to directly support a shift toward "information 

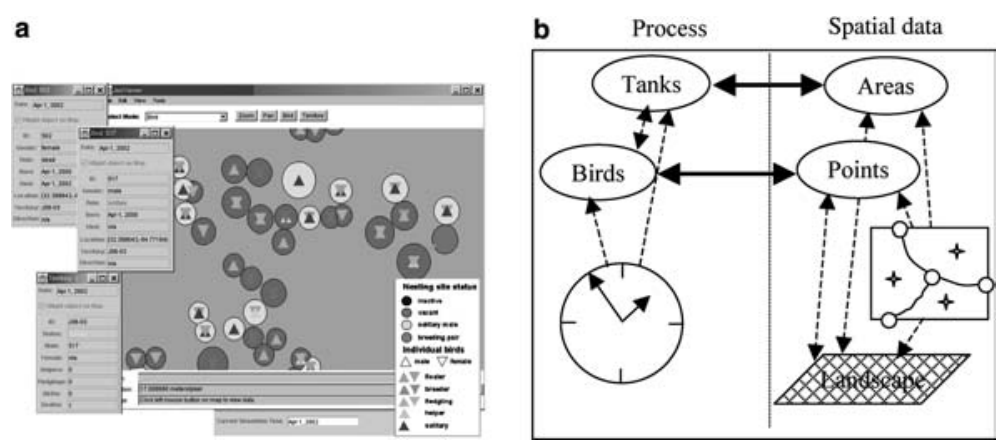

Fig. 5. The IDLAMS model. a RePast Interface. b Conceptual relationships between process and spatial data models

superiority and dispersed, networked force capabilities" within the US Navy (Clark 2002) by simulating military mobile communications in and around amphibious landing areas. TSUNAMI simulates battle space motion and interaction over real terrain data, applies rule sets to simulate message traffic, simulates quality of service protocols, and simulates exercise data management choices on a case-by-case basis, to model traffic loading using realistic tactical network architectures. The TSUNAMI simulation tracks the current state of global and local battle spaces (Fig. 4a)

In this model, all agents (including soldiers, tanks, jeeps, airplanes, and ships) have identity relationships with spatial features (i.e., points) (Fig. 4b). Attributes of the agents include location, current heading, fuel or battery levels, communications equipment, a communications routing capability that uses specific protocols, differing sensing capabilities, ranges of mobility, and sets of fuel and battery lifetimes. Causal relationships are described through the agents' abilities to move, to sense surroundings, and to communicate. Each of these basic behaviors has appropriate variations that add detail and fidelity. For example, agents move throughout the space in three different ways: (a) responsive motion to fight, follow, or flee other agents; (b) scripted motion defined by the model users before a simulation run; and (c) random motion when no other options are available. The agents move across the landscape and surrounding waterways and react to one another. The agents can change their attributes based on the other agents they encounter as well as the features of the terrain. For example, a tank may increase the power output of one of its radios if it is obscured in mountainous terrain but has a message for an allied aircraft circling nearby. Temporal relationships are handled within the model through asynchronous event scheduling. As each event occurs, the agents determine their new status and then update the GIS accordingly (i.e., asynchronously). Topological relationships are utilized when the agents query the GIS to determine the locations of neighboring agents and the type of terrain surrounding them. The model makes use of spatial analysis functions within the GIS to carry out these queries, the results of which are passed back to the model. By querying the landscape for allowable moves, the model makes use of topological rules that are coded within the ABM, but implemented with the help of GIS queries. A communications network topology is constructed by the agents (i.e., within the ABM) using selected self-configuring protocols. 
This model is implemented through a tight coupling of the RePast platform and the Java-based OpenMap GIS (http://openmap.bbn.com). The coupling takes an ABM-centric approach, in which GIS components were accessed through the ABM program. The grid-based landscape is currently handled within the ABM to avoid inefficiencies in converting this information back and forth from the GIS to the ABM. A more balanced integration of GIS and ABM could provide for better handling and updating of raster layers.

\subsection{Integrated dynamic landscape analysis and modeling system (IDLAMS)}

The Integrated Dynamic Landscape Analysis and Modeling System (IDLAMS) is an individual-based, spatially-explicit model of red-cockaded
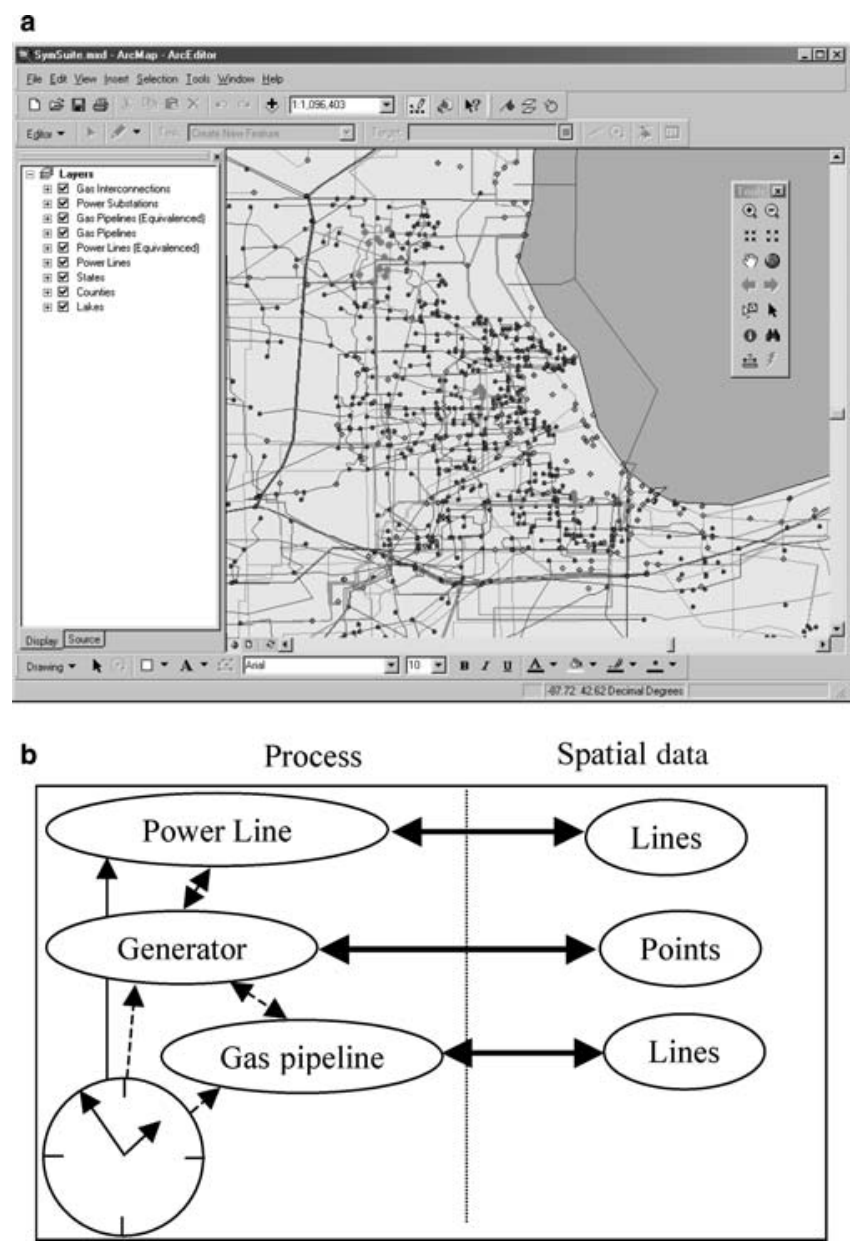

Fig. 6. The Infrastructure SymSuite. a ArcGIS interface, with electric power infrastructure locations, natural gas infrastructure locations and hypothetical critical components. The infrastructure information shown in the example is for illustrative purposes only. b Conceptual relationships between process and spatial data models 
woodpecker populations (Fig. 5a). The goal of this system is to integrate ecological models with decision support tools to allow military land managers and decision makers to balance mission preparedness with a diverse set of multiple land-use goals (Rewerts et al. 2000).In particular, there is a need to protect endangered species that find refuge from urban encroachment on military bases.

All agents in this model (i.e., representing individuals of the red-cockaded woodpecker [Picoides borealis] and pieces of military equipment) have identity relationships with spatial features (i.e., points for birds and areas for military equipment) (Fig. 5b). The causal relationships involve movement of birds and other agents and changes in the birds' attributes as they grow and are affected by other features and attributes. Military equipment (e.g., jeeps and tanks) affects the landscape as it moves across the terrain, including changing the suitability of habitat for the birds and, potentially, destroying nest sites. Movement of birds from one place to the next characterizes nesting, breeding and feeding behaviors, based on Letcher et al.(1998). For example, the birds can find open lands with appropriate trees for nesting and then choose these trees as nesting sites. This decision causes a change in an attribute of the agent (i.e., life cycle changes from non-nesting to nesting). This also affects a change in the raster landscape (i.e., from suitable nesting site to nest).Temporal relationships in the model are handled in the ABM asynchronously. The model reads initial environmental data in the form of a grid and handles this information within the ABM.The GIS is updated as events cause changes (i.e., asynchronously) to the attributes and locations of features and to attributes on the grid-based landscape.The history of the processes is not saved, but the last location and state are written to the GIS. Topological relationships, for example when a bird evaluates nearby or all locations for possible movement, and are handled exclusively within the ABM.

The model, like TSUNAMI, uses an ABM-centric tight coupling, using functionality from the GIS (i.e., Java-based JeoViewer developed at Argonne National Lab) in a model written with RePast. Like the other models, IDLAMS maintains and updates the grid-based landscape map using spatial analysis functions and asynchronous updating tools within RePast. Because the history of the process is not stored asynchronous updates to the GIS do not require time stamps, which would complicate the process. A more integrated approach could take advantage of GIS-based functions for updating and analyzing raster layers.

\subsection{Infrastructure simulation}

The goal of the infrastructure simulation suite (Infrastructure SymSuite) is to represent the operation of interdependent infrastructure systems such as electric power grids, natural gas systems and telecommunications networks (Thomas et al.2003). The system models the complex interplay between infrastructures to evaluate the effects of changes in one on the other and overall system vulnerability (Fig. 6a)

All agents in this model (including electric power lines, power generators, power demands, natural gas pipelines, gas sources, gas storage, gas consumption, and telecommunications systems) have identity relationships with spatial features (Fig. 6b). The feature attributes can change, but the 
features themselves do not move. All causal relationships, including effects of one agent on another, are implemented within the ABM. For example, the unexpected rupture of a natural gas main will cause a drop in pressure at the pipe's endpoints. This drop in pressure in turn will slow and then stop the delivery of natural gas to the destination endpoint of the pipe. This may in turn reduce or curtail the output of a natural gas-fired electric generator connected to the pipe's destination. The attributes within the GIS database of the gas main, pipe endpoints, and generator are updated by the associated agents. The $\mathrm{ABM}$ handles all temporal relationships, including asynchronous processes, but the agents update the GIS database synchronously at fixed intervals, to simplify the temporal data structure. The GIS database provides information on the initial topological relationships between all features. Any changes to the topology, e.g., a power line down, are modeled exclusively within the ABM and are not written back to the GIS because most such changes are temporary.

The model uses tight coupling, in which updates to the GIS database and the graphic interface are in real-time, as the model runs. The implementation uses middleware to manage the identity mapping between agent identifiers and spatial feature identifiers. In addition, it takes advantage of the fact that the GIS can store topological information, in addition to features' locations and attributes. The topological changes, as with all of the dynamics of the model, are integrated by the ABM, written with RePast. An earlier version of this model, based on ArcSDE, used versioning within the GIS to allow the ABM to store asynchronous, rather than synchronous, updates.

\subsection{Future middleware development}

A reasonable path for future development of such middleware is to further develop the causal, temporal, and topological relationships described above. For example, the application for which Infrastructure SymSuite was developed did not require that the agents and their associated features be able to move. An obvious extension is to allow the agents and their features to move. Moreover, topological connections between agents are read initially from the GIS and subsequently managed within the agent model. Another extension, then, would be to develop more dynamic links between the topology of the agents and the topology of the features. Similarly, many models use neighborhood calculations on a grid-based environment, some of which are implemented within the ABM. Because these calculations are common within GIS, it makes sense to implement them using the GIS platform. This extension is challenging because the common approach in GIS to performing a grid-based operation is to update the entire map simultaneously (i.e., synchronous updating). It is more efficient, in many cases, for an agent to update a cell value when needed, rather than updating all cells simultaneously. Finally, a means to communicate information about the clock of the ABM to the GIS, for the purposes of coordinating with agent-independent processes, if there are any, is needed.

In addition to functional extensions, the middleware approach could be written to accommodate multiple alternative GIS and ABM platforms. Such a general system requires a very clear specification of the generic architecture of these linkages, which is beyond the scope of this paper. 


\section{Conclusions}

The integration of spatial process and data models described in this paper can facilitate spatio-temporal inquiry into processes at the level of agents or individuals and data about specific cases and places. For example, incorporating real spatial heterogeneity into agent-based models will improve our ability to draw conclusions about the behavior of complex systems in realistic environments, which may be different from conclusions drawn with artificial environments. Model calibration and validation can be facilitated by providing a platform to test the broad outcomes of a model and the specific mechanisms that produce intermediate results (like the placement of a feature at a particular point in time). Specific claims of scenarios or predictions can gain acceptance when compared with data about specific places. Finally, descriptions of process afforded by ABM techniques can enrich the descriptions of form provided by GIS. For example, process descriptions in ABM could be used to facilitate spatial-temporal interpolation, to query a database for the location of feature/agent at a time in between two times stored in the database.

Object-orientation provides new opportunities for the integration of spatial process and data models, especially those that involve movement. While there are many ways to achieve such integration, we have advocated tight coupling of agent-based modeling and geographic information systems. We have discussed the kinds of relationships that need to be considered and implemented to achieve such coupling, and discussed issues and approaches to implementing these relationships. Among the possible approaches to tight integration, which include those we call ABM-centric, GIS-centric, and integrated approaches, we focused on an integrated approach that uses middleware to link existing GIS and ABM software systems. This approach provides a mapping from one system to the other, takes advantage of the past development efforts in object-oriented ABM and GIS software systems, and may be a reasonable short-term approach to implementing models that integrate sophisticated representations of form and process. A disadvantage is that the approach does not fully encapsulate spatial features and their behaviors. Among four example models that we present, one implements the middleware approach for simulation of infrastructure. Though we acknowledge that the middleware approach is not the only, nor necessarily the best, approach to implementing integrated GIS and ABM, it offers an approach that has practical advantages and should be pursued in parallel with other approaches.

\section{References}

Batty M (1997) Cellular automata and urban form: A primer. Journal of the American Planning Association 63(2): 266-274

Bell EJ (1974) Markov analysis of land use change: Application of stochastic processes to remotely sensed data. Socioeconomic Planning Sciences 8: 311-316

Benenson I, Torrens PM (2004) Geosimulation: object-based modeling of urban phenomena. Computers Environment and Urban Systems, 28(1-2): 1-8

Bian L (2003) The representation of the environment in the context of individual-based modeling. Ecological Modelling 159: 279-296 
Blaut JM (1961) Space and Process. The Professional Geographer 13(4):1-7

Brown DG, Pijanowski BC, and Duh J-D (2000) Modeling the relationships between land-use and land-cover on private lands in the Upper Midwest, USA. Journal of Environmental Management 59: 247-263

Brown DG, Page SE, Riolo RL, Zellner M, Rand W (In Press) Path dependence and the validation of agent-based spatial models of land-use. International Journal of Geographical Information Science,

Burks A, ed. (1970) Essays on Cellular Automata. University of Illinois Press, Champaign, IL

Burnham BO (1973) Markov intertemporal land use simulation model. Southern Journal of Agricultural Economics July: 253-258

Clark V (2002) Sea Power 21: Projecting decisive joint capabilities. Proceedings of the Naval Institute, U.S. Naval Institute, Annapolis, MD

Clarke KC, Brass JA, Riggan PJ (1994) A cellular automaton model of wildfire propagation and extinction. Photogrammetric Eng. and Remote Sensing 60(11): 1355-1367

Couclelis H (1997) From cellular automata to urban models: new principles for model development and implementation. Environment and Planning B: Planning and Design 24: 165-174

Cova TJ, Goodchild MF (2002) Extending geographical representation to include fields of spatial objects. International Journal of Geographical Information Science 16(6): 509-532

Deadman P, Gimblett RH (1994) A Role for Goal-Oriented Autonomous Agents in Modeling People-Environment Interactions in Forest Recreation. Mathematical and Computer Modelling 20(8): 121-133

DeAngelis DL, Gross LJ (1992) Individual-based Models and Approaches in Ecology : Populations, Communities, and Ecosystems. Chapman and Hall, New York

De Vasconcelos MJP, Goncalves A, Catry FX, Paul JU, Barros F (2002) A working prototype of a dynamic geographic information system. International Journal of Geographical Information Science 16(1): 69-91

Eastman JR (2001) IDRISI32 Release 2 [IDRISI for Windows Version 3.0]. Clark University, Worcester MA

Epstein JM, Axtell RL (1996) Growing Artificial Societies: Social Science from the Bottom Up. MIT Press, Cambridge, MA

ESRI (2004) ArcGIS v. 9.0. Environmental Systems Research Institute, Redlands, CA

Gahegan M (2003) Is inductive machine learning just another wild goose (or might it lay the golden egg)? International Journal of Geographical Information Science 17 (1): 69-92

Gardner M (1970) Mathematical games. Scientific American 223: 120-123

Gimblett RH (2002) Integrating Geographic Information Systems and Agent-Based Modeling Techniques for Simulating Social and Ecological Processes. Oxford University Press, New York

Goodchild MF (2002) Measurement-based GIS, in W Shi, PF Fisher, MF Goodchild (eds) Spatial Data Analysis. Taylor \& Francis, New York

Hägerstrand T (1967) Innovation Diffusion as a Spatial Process. University of Chicago Press, Chicago

Holland JH (1995) Hidden Order: How Adaptation Builds Complexity. Addison-Wesley, Reading, MA

Iglesias CA, Garijo M, Gonzalez JC (1999) A survey of agent-oriented methodologies. In JP Muller, MP Singh, AS Rao (eds), Intelligent Agents V-Proceedings of the Fifth International Workshop on Agent Theories, Architectures, and Languages (ATAL-98), Lecture notes in Artificial Intelligence. Heidelberg, Springer-Verlag

Kemp KK (1997) Fields as a framework for integrating GIS and environmental process models. Part one: Representing spatial continuity. Transactions in GIS 1(3): 219-234

Langran G (1992) Time in Geographic Information Systems. Taylor and Francis, Bristol, PA

Letcher BH, Priddy JA, Walters JR, Crowder LB (1998) An individual-based, spatially-explicit simulation model of the population dynamics of the endangered red-cockaded woodpecker, Picoides borealis. Biological Conservation 86: 1-14

Leung Y, Leung KS, He JZ (1999) A generic concept-based object-oriented geographical information system. International Journal of Geographical Information Science 13(5): 475-498

Liu XH, Andersson C (2004) Assessing the impact of temporal dynamics on land-use change modeling. Computers Environment and Urban Systems, 28: 107-124 
Liverman D, Moran EF, Rindfuss RR, Stern PC (1998) People and Pixels: Linking Remote Sensing and Social Science. National Academy Press, Washington, DC

Maidment DR (1996) Environmental modeling within GIS. In: MF Goodchild, LT Steyaert, BO Parks, C Johnston, D Maidment, M Crane and S Glendinning (eds) GIS and Environmental Modelling: Progress and Research Issues. Fort Collins, GIS World. pp. 315-324

Minar N, Burkhart R, Langton, C, Askenazi M (1996) The Swarm simulation system: A toolkit for building multi-agent simulations. Working paper Santa Fe: Santa Fe Institute

Parunak HVD, Savit R, Riolo RL (1998) Agent-based modeling vs. equation-based modeling: A case study and user's guide. Proceedings of Multi-agent systems and Agent-based Simulation (MABS'98), Springer

Peuquet DJ (2002) Representations of Space and Time. Guilford Press, New York

Peuquet DJ, Duan N (1995) An event-based spatiotemporal data model (ESTDM) for temporal analysis of geographical data. International Journal of Geographical Information Systems 9(1): $7-24$

Rand W, Brown DG, Page SE, Riolo R, Fernandez LE, Zellner M (2003) Statistical validation of spatial patterns in agent-based models. Proceedings, Agent Based Simulation, Montpellier, France, April 2003

Raper J, Livingstone D (1995) Development of a geomorphological spatial model using objectoriented design. International Journal of Geographical Information Systems 9(4): 359-383

Rewerts CC, Sydelko PJ, Dolph JE, Shipiro AM, Taxon TN (2000) An object-oriented, individual-based, spatially explicit environmental model: A discussion of the approach to implementing the system. $4^{\text {th }}$ International Conference on Integrating GIS and Environmental Modeling (GIS/EM4): Problems, Prospects and Research Needs, Banff, Alberta Canada. Sept. $2-8,2000$

Ropella GE, Railsback SF, Jackson SK (2002) Software engineering considerations for individual-based models. Natural Resource Modeling 15(1): 5-22

Sheynin OB (1988) A A Markov's work on probability. Archive for History of Exact Science 39: $337-377$

Thomas WH, North MJ, Macal CM, Peerenboom JP, (2003) From physics to finances: Complex adaptive systems representation of infrastructure interdependencies. Naval Surface Warfare Center Technical Digest, Naval Surface Warfare Center, Dahlgren, VA pp. 58-67

Tobler WR (1970) A computer movie simulating urban growth in the Detroit region. Economic Geography 46(Supplement: Proceedings, International Geographical Union. Commission on Quantitative Methods): 234-240

Tobler W (1975) Linear operators applied to areal data. In: JC Davis, MJ McCullagh (eds) Display and Analysis of Spatial Data, North Atlantic Treaty Organization. Scientific Affairs Division., Kansas Geological Survey. and University of Nottingham. Dept. of Geography. London, Wiley and Sons, New York, pp. 14-37

Tobler WR (1979) Cellular geography. In: S Gale, G Olsson, D Reidel (eds) Philosophy in Geography. Kluwer, Dordrecht, pp. 379-386

Toffoli T, Margolus N (1987) Cellular Automata Machines: A New Environment for Modeling. MIT Press, Cambridge, Mass

Tomlin CD (1990) Geographic Information Systems and Cartographic Modeling. Prentice Hall, Englewood Cliffs, NJ

Torrens PM, Benenson I (In Press) Geographic automata systems. International Journal of Geographical Information Science

Turner BL, Skole D, Sanderson S, Fischer G, Fresco L, Leemans R (1995) Land-Use and LandCover Change Science/Research Plan. Joint publication of the International GeosphereBiosphere Programme (Report No. 35) and the Human Dimensions of Global Environmental Change Programme (Report No. 7). Royal Swedish Academy of Sciences, Stockholm

Ulam S (1962) On some mathematical problems connected with patterns of growth of Figures. Applied Mathematics 14: 215-24

University of Chicago (2003) RePast 2.0. (Software). Chicago: Social Science Research Computing Program. Available at http://repast.sourceforge.net

Van Duersen WPA (1995) Geographical Information Systems and Dynamic Models. PhD Thesis, Faculty of Spatial Sciences, University of Utrecht. Utrecht, The Netherlands 
Verburg PH, de Koning GHJ, Kok K, VeldKamp A, Bouma J (1999) A spatial explicit allocation procedure for modeling the pattern of land use change based upon actual land use. Ecological Modelling 116: 45-61

Von Neumann J (1966) Theory of Self-Reproducing Automata. Edited and completed by A. W. Burks. University of Illinois Press, Urbana, Illinois

Wachowicz M (1999) Object-oriented design for temporal GIS. Taylor and Francis

Wesseling CG, Karssenberg D, Van Deursen WPA, Burrough PA (1996) Integrating dynamic environmental models in GIS: the development of a Dynamic Modelling language. Transactions in GIS 1: 40-48

Westervelt JD, Hopkins LD (1999) Modeling mobile individuals in dynamic landscapes, International Journal of Geographical Information Science 13(3): 191-208

Worboys MF (1994) A unified model of spatial and temporal information. The Computer Journal, 37(1): 26-34

Yuan M (2001) Representing complex geographic phenomena in GIS. Cartography and Geographic Information Science 28(2): 83-96.

Zeigler BP, Praehofer H, Kim TG (2000) Theory of Modeling and Simulation: Integrating Discrete Event and Continuous Complex Dynamic Systems, $2^{\text {nd }}$ Edition. Academic Press, New York 